\title{
African burden of Mental Health: necessity of global exchange between researchers
}

\author{
Etindele Sosso FA $^{1^{*}}$, Nakamura $\mathbf{O}^{2}$ and Mitsu $\mathbf{N}^{3}$

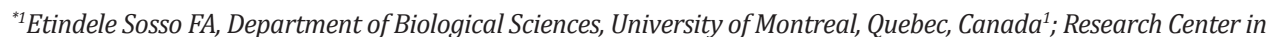 \\ Neuropsychology and Cognition, Quebec, Canada ${ }^{2}$ \\ ${ }^{2}$ Nakamura O, Global Research Department, Ritsumeikan University, Kyoto, Japan ${ }^{3}$ \\ ${ }^{3}$ Mitsu N, The graduated University of Advanced Studies, Miura, Japan ${ }^{4}$
}

Received: 10 October, 2017; Accepted: 01 November, 2017; Published: 06 November, 2017

*Corresponding author: Etindele Sosso FA, Department of Biological Sciences, University of Montreal, Quebec, Canada ${ }^{1}$, Research Center in Neuropsychology and Cognition, Quebec, Canada², Postal address: 90, avenue Vincent d'Indy, H2V2S9, Tel: +1 514343 6111 \#3187; Email: faustin. armel.etindele.sosso@umontreal.ca

\section{Introduction}

Administration of global health in low- and middle- incomes countries faced several barriers, mainly because governments considered only tropical and metabolic diseases asmajor's public health matters. The prevention and mastering of the mental health as well as side effect linked to environment of population (1-11)are the best examples. Recent published articles showed that, neurodegenerative diseases as well as dementia; was not handled like the other diseases like aids and malaria. Lake of research around lifestyle risk factors in lowand middle- income countries, and new discoveries in mental disorders(1-5), may induces this important difference between developing countries and the rest of the world. Many countries concerned by this difference were in Africa and South America (11). Several authors reported findings about mental disorders in South America. In the other hand, most of publications related to health in Africa, even in high-impact factors journals, only reported findings regarding tropical diseases or infectious diseases. When looking through literature about global health, except north hemisphere countries, current literature always shows a better picture of populational health in South American compared to Africa(11). Our opinion is the possibility that, this inequality in literature emerged from a deficit in research in african countries. Structure for research, investments and program related to research, are almost absent. Health promotion in the continent was subsequently improved during the last twenty years, because of massive initiative and funding. Global health intervention were numerous and continuous in the field of mother and child health, cardiometabolic syndromes, and infectious diseases. The mental health is the sector of health in Africa, where expenditure and programs does not really exist or are very fewer compared with the management of diseases above. This situation leads to a difference between north and sub- saharian African countries. The proximity between Maghreb and Europe, and the close collaboration between their researchers allows a permanent update of north african health system. The local health administrator understands importance of research despite the absence of funding, and encourages researcher with international training and symposium, and contributes in open access publication often. The same actions should be made for the rest of Africa, beginning with sharing information between local researchers, and increase of continental congress. Many private funding dedicated to african researchers are promoted by private donators like Bill and Melinda Gates foundation, but information is not well share through network or academician world and eligibility criteria for researchers based in the continent are discriminative. They are judge with the same scale of productivity than their European and American colleagues. Only few of them met conditions of these funding and finally majority of winners are based outside Africa, and then led research without really knows the continent. The necessity of global exchange between researchers of both hemisphere are an emergency.

\section{Conclusion}

The world is moving, so culture and health administration should follow at the same speed. Before thinking individually, think global. Plans and prevents the potential impact of a common action made by researchers and supports by politic in the following decades. There is a difference between north and south hemispheres, but also between low-and middle- income countries; in terms of publications and research. Africa has intellectual resources to lead very good research projects and provide publications in high impact factors journals, but without an exchange with internationals experts in the field and without appropriate supports of their own countries; the difference will continue to increase. 


\section{References}

1. Etindele Sosso FA, Nakamura O, Nakamura M. Evaluation of Combined Effects of Insomnia and Stress on Sleep Quality and Sleep Duration. J NeurolNeurosci. 2017;8:3. doi: 10.21767/2171-6625.1000202

2. Etindele Sosso FA. Negative Involvement of the Working Environment in the Occurrence of Cognitive Disorders. Transl Biomed. 2017;8:2. doi:10.2167/2172-0479.1000109

3. Raouafi S, Etindele Sosso. Cyberpsychology: Video Games as a perspective for Cognitive Training. Ment Health Addict Res. 2017;2:3,1-2. doi:10.15761/MHAR.1000141

4. Etindele Sosso FA. Sleep disorders and insomnia: effects on a young population. Psychology and Psychiatry. 2017;2:1, 26-32.

5. Etindele Sosso FA. Neurocognitive game between risk factors, sleep and suicidal behaviour. Sleep Sci. 2017;10:1,41-46. doi: 10.5935/19840063.20170007
6. Etindele Sosso FA, Raouafi S. An overview of Positive Interaction between Exercise and Mental Health. J NeurolNeurosci. 2017;8:4

7. Etindele Sosso FA, Raouafi S. Brain disorders: correlation between cognitive impairment and complex combination. Ment Health Fam Med. 2016;12(2):215-222

8. Etindele Sosso FA, Raouafi S.Appropriate sleep duration and physical activity modulate cognitive improvement. J Sleep Disor. 2016; 5:4. doi: 10.4172/2325-9639.1000182

9. Etindele Sosso FA, Hito MG, Bern SS. Basic activity of cortical neurons during somnolence induced by anesthesia. J NeurolNeurosci. 2017;8:4. doi:10.21767/2171-6625.1000203

10.Etindele Sosso FA. Visual dot interaction with short-term memory. Neurodegener Dis Manag. 2017;7(3):183-190. doi: 10.2217/nmt2017-0012

11.Etindele Sosso FA, Kaboré P. The African burden of mental health. J Ment Disord Treat. 2016;2-2. doi: 10.4172/2471-271X.1000122 